\title{
The anatomy education during Covid 19 and the future challenges
}

\begin{abstract}
The COVID-19 pandemic has had profound impacts on medical education globally. Worldwide, most medical students have missed months of educational experience that is considered fundamental in their training. Advances in technology are a potential solution to the problem. Forms of interactive learning, use of online platforms, as well as variants of e-lecture teaching are considered as alternatives to in-person teaching, as a part of a modernized anatomy curriculum. In addition to the already widespread online meeting platforms, we also discuss the use of video recordings with integrated slides, real pictures and short videos which can be uploaded onto the university platform Moodle. Alternatives to dissections, prosections, plastination, microscopy and videos are considered as potential forms of organization in the learning process. In regards to dissections, the problem of acquiring practical skills in an environment of social distancing and the successful accomplishment of tests and exams is also discussed.
\end{abstract}

Keywords: anatomy education, COVID-19, learning approaches
Volume 6 Issue 4 - 202।

\author{
Maslarski I,' Stoikov V,' Ingilizova $\mathrm{G}^{2}$ \\ 'Departmant of Anatomy, University of Sofia, Bulgaria \\ 2Vita" Multidisciplinary Hospital for Active Treatment, Bulgaria
}

Correspondence: Ingilizova G,Vita" Multidisciplinary Hospital for Active Treatment, Sofia, Bulgaria, Tel +359887204480, ORCID 0000-0002-07I8-458I,Email ingilizova@mail.bg

Received: June 31, 202I | Published: July I5, 202 |

\section{Introduction}

The current worldwide outbreak of COVID-19 (the disease caused by Severe Acute Respiratory Syndrome Coronavirus 2 (SARSCOV-2) has had a detrimental effect on the socio-economic life of people like no other event in this generation's history. The disruptions in the educational process that we are facing are imposing a serious review of the pedagogic practice, incorporating new and necessary methods in the anatomy, histology and cytology curriculum in particular. Current advances in technologies are enabling us to use online teaching platforms, and interactive educational softwares making e-learning possible. For a short period of time, the education system has been severely tested, both in primary and secondary schools and in universities. Unscripted events, like the coronavirus crisis, are what illustrate a university's core values. It is essential to use a complete resource of available teaching methods, interdisciplinary links combined with innovative interactive techniques. ${ }^{1}$ The training of university students with a specific professional orientation (medical students in particular), requires developing a specific practical skills and competencies. This makes the situation even more complex and almost without alternatives.

Medical education (the initial training to become a physician and the additional training thereafter - residency, fellowship) belongs to the so-called "regulated professions", which implies that access and exercise is subject to the possession of a specific professional qualification and skills. This in a way excludes the possibility of a distance education, at least not exclusively, as would be the case in other professional fields. The anatomy curriculum is one of the pillars in the medical education and takes a great place in the shaping of future physicians. In this article we will present a theoretical model of education, the main object of which is to prevent the spread of the Covid19 infection whilst maintaining a high quality education. Following basic principles, to ensure enough time is devoted for the formation of practical skills that could not be given in a distant learning only. We have created a hybrid educational model that combines online educational materials with traditional place-based methods. This was achieved by using selected software products, such as digital online microscopy and video lectures. The incorporation of online platforms into everyday teaching was highly approved by the students, because it made the educational process more personalized.

Early on it was very clear that the most challenging aspect of teaching anatomy online would be the inability of the students to participate in anatomical dissections, one of the traditional ways of learning anatomy. We modified the practical classes, by putting more emphasis on prosections and clear presentation of key anatomical structures. In this way, we both ensured physical distancing and we increased the productivity and efficiency of the class. The educational disruption was set off by series of chronological events. On March 8, 2020 the first positive case of Covid19 in Bulgaria was confirmed. This was followed by an order of the Rector of Sofia University "St. Kliment Ohridski" to suspend all attending classes. On March 13, 2020, the Minister of Health issued an order for an emergency epidemiological situation and suspended all in-person classes indefinitely in all educational facilities.

Every university in the country had to come up with a strategy for distant learning in order to continue with the educational process. The Faculty of medicine at the Sofia University reached a conclusion that every department would have to decide about the educational modus separately, and in accordance with the needs of the curriculum. In contrast to other medical faculties in the country, e-learning was not seen as sufficient for finishing the semester successfully, guaranteeing the required anatomical knowledge.

The complete suspension from attending classes lasted three months, after which an intensive in-person training course was held in all disciplines. That's one of the main reasons that the decision to return to in-person teaching was made as soon as it was deemed safe. In this article, we would like to share our experience during the educational process in the human anatomy course, as well as histology of the human organs, which took place in two stages: The first during the online learning and the second one during the intensive in-person teaching. We will also share our plan for the future in a circumstances of ongoing epidemiological situation. 


\section{Materials and methods}

The above mentioned chronology of events, led us to the theoretical planning of a new model of training, which would maintain a reasonable and decent level of education, while complying with the anti-epidemic measures.

For this purpose, a hybrid educational model was conceptually constructed by the Anatomy, Histology, Pathology and Forensic medicine department. An innovative method was used to conduct online lectures, by pre-recording videos with high-quality images and audio explanations, creating and sharing virtual, live meetings etc. The practical exercises were replaced by a virtual microscope, light microscopy photographs and electronograms. The knowledge assessment of the students was carried out through various questionnaires, tests about recognizing essential anatomical structures on microscopic images and the ability to present them in an electronic environment and discussion.

\section{Results}

We measured the effectiveness and quality of the e-learning course by looking at the results from the colloquia and test conducted during the summer semester. We chose them in particular because they specifically measure the knowledge and skills of the students, acquired during the hybrid method. We compared the results with the results from the previous three years, during which the students were educated only with the traditional place-based classroom methods. For example, the average grade from the colloquium for digestive and respiratory systems is 4.65 , with a standard deviation of 0.2 ; The average grade for the same colloquium conducted in hybrid-education is 4.72; The difference was not seen as significant; We are fully cognizant and do not claim that we have conducted a comparative study with a great statistical and scientific value, given that we used the results from the previous year, which were not intended as such. Regarding the other two colloquia for the internal organs, the data are similar and show a minor difference in the final results. The colloquia are practical and use evaluation criteria, such as: microscopy, describing histological structures, making casual relationships between macroscopic and microscopic anatomy, naming anatomical structures on cadaveric organs and understanding the clinical significance and relations.

\section{Discussion}

Although we fully agree that the major concern in these unprecedented times are patients and communities, we should also protect these generation's education. We wouldn't be surprised if medical education changes permanently, and adequately prepared physicians are more needed than ever. When the first Covid19 patient was diagnosed and we were forced to continue our education online, we were surprised and unprepared. We reacted almost immediately with a major package of digital measures. This proved to be a serious challenge in terms of practical classes, as we couldn't substitute some of the basic methods and techniques in anatomy, for example microscopy and cadaver dissection, being the modus-operandi in anatomy for centuries. ${ }^{2-4}$ After a week of complete interruption of the educational process and numerous online councils within the department, the decision was made to restructure part of the educational process in an online fashion. Initially we started using the platform Moodle, which is the official online learning platform of the University of Sofia. This platform has numerous advantages as you could create and share virtual halls with students, give assignments and test, and check their activity online. This was however counteracted by the disadvantages we faced early on. A lot of the issues that we encountered were technical - broadcasting delays, audio problems and the inability to prerecord materials for repeated use.

The bigger problem we faced during the time was the practical course, as we couldn't find an answer to the question, can we find a substitute for something long-established? The digital platform we were using at the time, Moodle, was great and suitable for diagnosis, but not so much for the formation of new knowledge, or even less for development of new skills. This led us to a revision of our established teaching approach, as we introduced the use of pre-recorded video lectures. We used a free software available on Google and Gmail, which allows you to create high-quality videos with acceptable length that we could integrate with platforms already available to us like Moodle and YouTube. We chose Screencast Recording for Gmail in particular, because other popular platforms were suitable for live connection only and did not allow video recording. This software proved to be very convenient, because besides the voice record option, we also had the advantage of presenting slide shows, integrate other videos from different sources, like world-renowned and leading experts in the field and all that, at just one click away. This proved to be very time-consuming and demanding for the teaching stuff, but also led us to creating a product for long term use, which the students could also access on their own and when they need to. This led to us incorporating the strategy of watch-read-repeat, as we could present new material to the students, they could catch up on theoretical materials regarding the issue, and could lastly visit the video materials on their own when needed. The final product was then uploaded on the university platform Moodle, as well as on YouTube with a private link, which could be then sent to the students at a certain day and time. In terms of duration, our goal was to keep the length of E-learning materials equal to that of in-person lectures. The created product consisting of pre-recorded lectures can be used in the future as a part of a modernized and individualized anatomical course (Figure 1).

As for student evaluation, the used software allows monitoring of student activity as each student signs in with his own personal username (using his student ID number) and password. It even creates statistics of the attendance, and registers how many minutes of the video have been watched. The downside is that we can't know for sure, if the student has launched the lecture without actually watching it. We overcame this problem with a direct audio connection available to us, at the beginning and at the end of the lecture. As described, the length of the lecture with discussion included before and after it, are in accordance with the duration of in-person lecture.

For the histology practical course, we used a mix of in-house made materials and online learning materials. This way, the students had access to a high quality microscopic slides, that they could manipulate in different ways. They allow increasing and decreasing the magnification to the next preset level, and relocation of the field to different observation areas (Figure 2). This figure shows the source we used for histology of internal organs. We also incorporated a lot of our own microscopic slides in the department, by using photographs, edited with attached markings on the different anatomical structures. In this way students acquire real skills for studying a histology slide, recognizing common essential structures, their relationship, position and communication. As follows, students create a connection between the macroscopic and microscopic structures of an organ, fully appreciating the organ's anatomical structure. They build a long lasting knowledge, a guiding star to lead the way in the medical future. A major disadvantage of such computer-based training, is the inability to form proper microscope skills. The digital change of magnification and change of position of the observed field is as close as possible to 
the real images, but cannot mimic it completely. It should be noted that even the best photos and histology atlases cannot compensate, and lead to the same results in one's education, as a real microscopy does. We also evaluated the learning progress of the students, by giving the different tasks on the slides. We encouraged them to develop their skills for detecting different structures and interconnected components, such as cells, fibers, blood and lymph vessels, glands etc.
For this purpose we used a free software, imitating a real microscope, asking them to point and mark certain anatomical structures. After each class, the students were obliged to write a protocol with drawn histological slides and their labelings. The completed protocols were then uploaded to the online platform - Moodle. After the protocols were checked by the assistants, feedback was provided to the students in the so-colled Moodle virtual rooms or Big blue button (BBB).

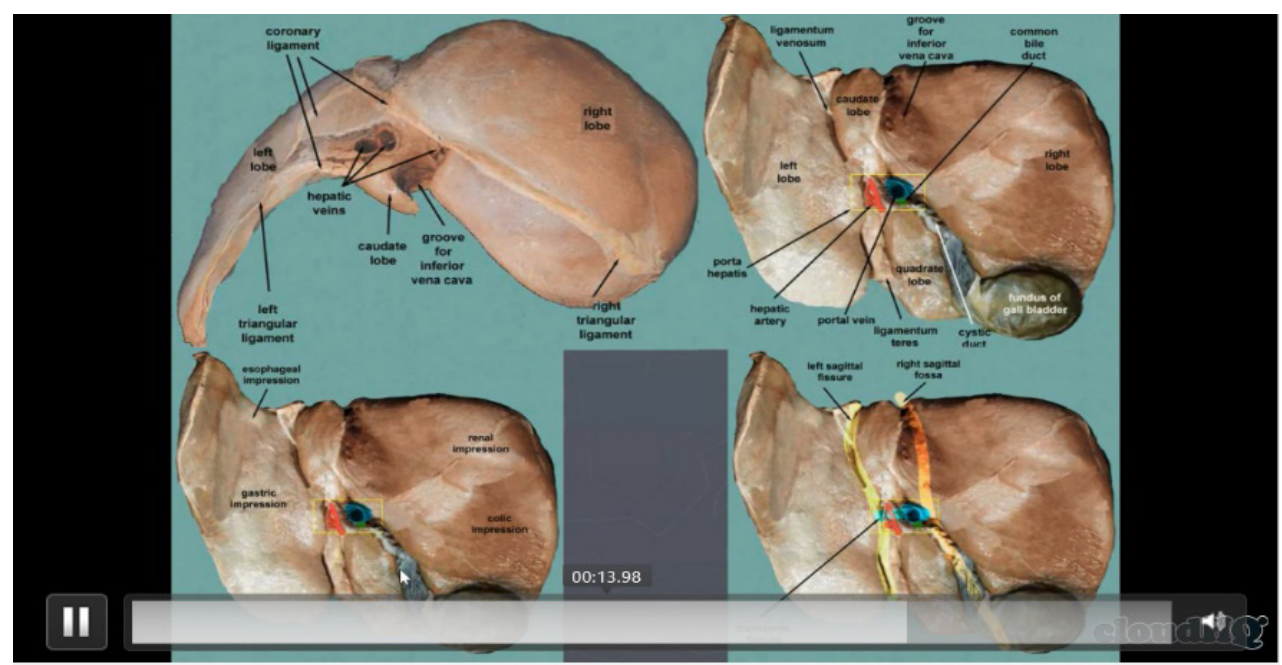

here to search

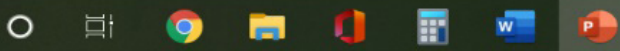

Figure I Screenshot of a video resource used for macroanatomy training.

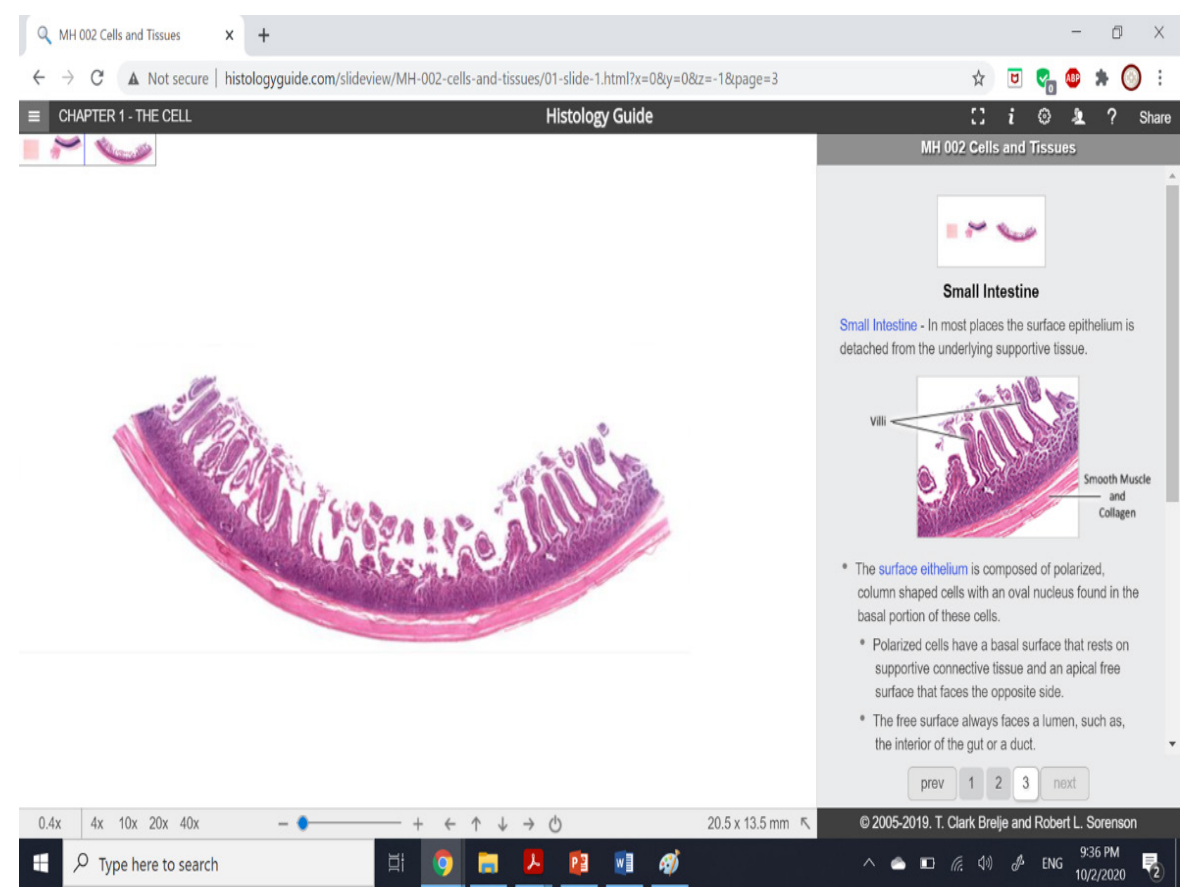

Figure 2 Screenshot from a microanatomy training resource.

Early on it was very clear that the most challenging aspect of teaching anatomy online would be the inability of the students to participate in anatomical dissections., ${ }^{5,6}$ Once again we relayed on videos and digital atlases. The teaching assistants often held online seminars and discussions with the students. It was clear to us that this could not completely replace the actual conduct of in-person anatomical classes and certainly could not create technical skills by the process of cadaver dissection. In the online program, we mainly relied on 3D models and videos recorded by the teaching assistants, explaining the topographical anatomy of the human body, the neurovascular bundles, 
the deep and superficial layers and their clinical significance. We used one of the basic tools in pedagogy, demonstration and professional conversation. No matter how desperately we tried to completely substitute classical way of learning anatomy, we understood that no form of e-learning could substitute the practical knowledge acquired in the anatomical theatre. That's one of the main reasons that the decision to return to in-person teaching was made as soon as it was deemed safe. Teaching was organized in such a way that we put an emphasis on the practical courses. These courses were carried out in the span of 2 months (June and July) in the form of intensified learning to compensate for lost time. It was decided that students would work in smaller groups of 12-15 people in the dissection hall with large area. We focused mainly on prosections and clear presentation of key anatomical structures by the assistant, with the students watching the activity from safe distance. After the prosections, the students would work in couple of smaller groups carrying out different activities. One group would dissect blood vessels and nerves, second group would examine previously extirpated internal organs, and others graphicly depict anatomical schemes under the guidance of an assistant or demonstrator. Every 20 minutes the students would switch between activities. It was decided that the total time for each class was to be no more than $2 \mathrm{~h}$.

The histology classes were organized in such a way, that the students concentrate on practical skill of microscopy. First, with the available technology in the department the teaching assistant would present new histological slides through a microscope connected to a camera, visualized on a screen. After which the students watch different slides independently, on different microscopes keeping safe distance from each other. All slides would be photographed and uploaded to a pre-created vitual room in Moodle, so it can be visible to all, and it can provide opportunity for discussion. The emphasis here is put on developing skills for microscopy, which cannot be formed otherwise. All sanitary and preventative measure were seriously enforced in order to prevent virus transmission - that included washing of hands, mandatory mask wearing, proper ventilation of the anatomical rooms.

For the upcoming academic year 2020-2021 our department is prepared for two different scenarios. Also, we are anticipating a transition from one to the other if necessary. The most feasible scenario is to combine the two methods of teaching - online and in-person, in a so-called hybrid manner. Of the total number of lectures, $2 / 3$ will be prepared and presented in an online fashion, with $1 / 3$ being still in-person in the form of seminars. The practical part of the anatomy course will be focused on prosections and clear presentation of key anatomical structures, and observing with the described rotational mechanism with the students divided into smaller groups. With the described organization, we hope to both ensure physical distancing, and also to increase the productivity and efficiency of the class.

The second plan, represent our strategy for organizing the educational process of teaching anatomy in the presence of a full ban of in-person teaching. In this case we would use the already implemented and tested methodology of e-learning. In such circumstances we will also provide an opportunity for revisiting the lost opportunity for generating specific anatomical knowledge and skills in the next semester or year. The anatomy curriculum covers two years of the entire academic process, which, thankfully, would allow us to disperse the material and catch up with practical classes in the next semester or year.

\section{Conclusion}

The planning, organization and implementation in anatomy teaching in an environment of a long lasting epidemic, present a serious challenge. With this report we are sharing our experience in a different form of battle against the SARS-CoV-2, the one in the dissection hall and histology lab. Trying to answer a great question, how do we keep our students and teachers safe and healthy, without sacrificing the quality of education. We focus on the non-traditional teaching tools, such as the described video-presentation lectures, the use of virtual microscopes and creating a specific work algorithm for the dissections. We measured the effectiveness and quality of the e-learning course by looking at the results from the colloquia and test conducted during the summer semester. We compared the results with the results from the previous three years, during which the students were educated only with the traditional place-based classroom methods. The difference was not seen as significant; We are fully cognizant and do not claim that we have conducted a comparative study with a great statistical and scientific value, given that we used the results from the previous year, which were not intended as such. A key feature of human nature is adaptability. Although these times of crisis have taken us to the brink of collapse, the methods of teaching we have developed and incorporated in the past few months could stay long after the Covid-19 pandemic has passed. It won't be a surprise if this plays the role of a catalyst in the process of evolution in the field of education. Our proactive response puts an emphasis on the advances in technology, in conjunction with the new and necessary alternative teaching methods. The right question to ask is not "Why?" but "How?". How do we keep the ball rolling after the crisis has passed and how do we incorporate the lessons learned today for tomorrow?

\section{Acknowledgments}

None.

\section{Funding}

None.

\section{Conflicts of interest}

None.

\section{References}

1. Rabjot R, Rafik S, Matthew P, et al. Social media and cadaveric dissection: a survey study. Clinical Anatomy. 2019;32(8):1033-1041.

2. Broadbent J, Poon W. Self-regulated learning strategies and academic achievement in online higher education learning environments: a systematic review. Internet High Education. 2020;27:1-13.

3. Pather N, Blyth P, Chapman JA, et al. Forced disruption of aatomy education in Australia and New Zealand: an acute response to the Covid-19 pandemic. Anat Sci Educ. 2020;13(3):284-297.

4. Kuldeep S, Shival S, Abhishek B, et al. Medical education during the COVID-19 pandemic: a single institution experience. Indian pediatrics. 2020;57(7):678-680.

5. Joe I, Marios L, Aaron SD, et al. A review of anatomy education during and after the COVID-19 pandemic: revisiting traditional and modern methods to achieve future innovation. Clin Anat. 2021;34(1):108-114.

6. John CM, John B, Paul B, et al. Teaching anatomy without cadavers. Medical Education. 2004;38(4):418-424. 